\title{
People as a Service: a mobile-centric model for providing collective sociological profiles
}

\author{
Jose Garcia-Alonso $^{1}$, Javier Miranda ${ }^{2}$, Javier Berrocal ${ }^{1}$, Juan Manuel Murillo ${ }^{1}$, \\ and Carlos $\mathrm{Canal}^{3}$ \\ 1 Department of Information Technology and Telematic Systems Engineering, \\ University of Extremadura, Spain \\ \{jgaralo, jberolm, juanmamu\}@unex.es \\ 2 Gloin, Calle de las Ocas 2, Caceres, Spain \\ \{jmiranda\}@gloin.es \\ 3 Department of Computer Science, University of Málaga, Spain \\ canal@lcc.uma.es
}

\begin{abstract}
Mobile devices have become increasingly popular in the everyday life of many individuals. By taking an insight into the most common uses of mobile devices we clearly appreciate that accessing internetbased services has grown greatly. This, and the fact that they are extremely personal gadgets has turned them into the main interface used by individuals to express themselves towards the outside world and to receive information from others. As a result of the highly personal use, mobile devices have been granted the potential to become unrivaled devices for building and storing the virtual profiles of their owners. Access to such profiles is of great interest in fields such as governance, health, smart cities, etc. Generating a centralized profile of a user is a task upon which a lot of interest has been put in the field of social mining. Peopleas-a-Service (PeaaS) is a computing model that seeks to establish the foundations upon which technologies that rely on mobile-centric computing models for social purposes should evolve.
\end{abstract}

Keywords: Sociological profiles, Mobile devices, People as a Service

\section{The Four Pillars of PeaaS}

PeaaS aims to exploit the potential of mobile devices for storing sociological profiles of their owners, and offering them as a service. This is an alternative to traditional social computing models where users explicitly generate information that is finally centralized in servers that stand beyond their control. The model has been conceived upon four pillars describing the basic considerations which must be complied in order to guarantee that it is correctly put into practice.

Mobile devices as interfaces to people. The PeaaS model understands mobile devices as interfaces to people, which connect them to the outside world and through which they express their thoughts and concerns. This makes mobile devices a centerpiece of the PeaaS computing model. 


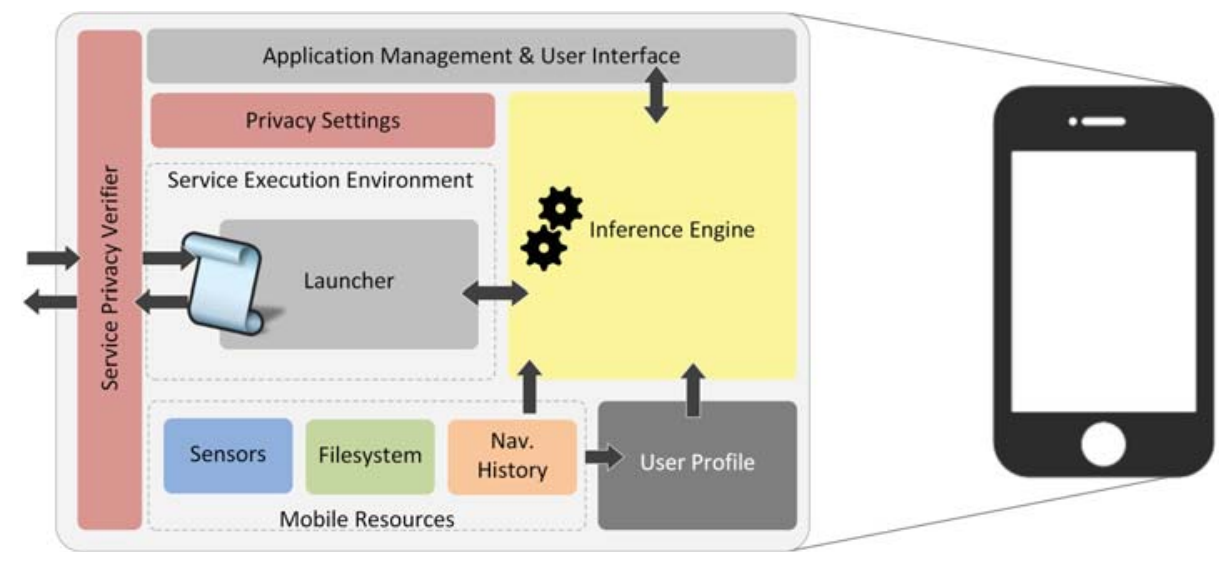

Fig. 1. High level reference architecture for PeaaS.

Sociological virtual profiles. Most mobile devices are equipped with a pack of sensors capable of collecting contextual information about their surroundings, and ergo also their owner's surroundings. The PeaaS model allows a sociological virtual profile of device owners to be built and kept in their device. Such profile is elaborated from the contextual information captured by the device's sensors and from the owner's interaction with the device.

Sociological profiles as a service. Generating profiles of the device owners is only useful to external entities if interactions with these profiles are enabled. PeaaS determines that profiles have to be provided as a service to those who may be interested in accessing this information.

User privacy. The PeaaS model guarantees that an individual's personal profile is always kept exclusively in his or her device. Furthermore, PeaaS makes all accesses to the information found within the devices completely visible and controllable by their owners.

These four pillars are combined in a mobile-centric computing model to overcome the limitations of current social computing models. Serving individuals' virtual sociological profiles through mobile devices goes one step beyond other models that focus only on providing the devices' contextualized data as a service. A reference architecture for PeaaS is shown in Figure 1 complying with the pillars described.

This paper was originally published in the IEEE Software journal and can be accessed in http://dx.doi.org/10.1109/MS.2013.140.

\section{Acknowledgments}

This work has been partially funded by the Spanish Government under Projects TIN2011-24278, TIN2012-34945 and TIN2012-35669. It has also been funded by the Government of Extremadura and FEDER funds, and by the University of Málaga, Campus de Excelencia Internacional, Andalucía Tech. 